\title{
FTIR Determination of Surfactant Removal from Arc Discharge Buckypapers for Air Sampling
}

\author{
Jacob S. Shedd ${ }^{1}$, Evan L. Floyd ${ }^{2}$, Jonghwa Oh${ }^{1}$, Claudiu T. Lungu ${ }^{1}$ \\ ${ }^{1}$ Department of Environmental Health Sciences, University of Alabama at Birmingham, Birmingham, AL, USA \\ ${ }^{2}$ Department of Occupational and Environmental Health, University of Oklahoma, Oklahoma City, OK, USA \\ Email: clungu@uab.edu
}

\begin{abstract}
In our previous work, buckypapers (BPs) were fabricated for use as sorbents for the air sampling of vapors. The fabrication methods used suspended arc discharge (AD) single-walled carbon nanotubes (SWNTs) in a surfactant and water solution, then subsequently vacuum filtered the suspension to remove the supernatant. The BPs were then heat-treated to remove any residual surfactants remaining from fabrication, which was corroborated by gravimetric and surface analysis. The present work sought to validate these findings using Fourier-transform infrared spectrometry (FTIR) analysis of heat treated (HT) and non-heat treated (NHT) BPs. The effect of humidity on FTIR spectra was also considered to determine proper storage of BPs. This was assessed by storing BPs in three different environments over the course of four weeks and performing FTIR analyses weekly to determine changes due to humidity. The findings of FTIR analyses appear to indicate surfactants were removed in the heat treatment process, though water interference in the spectral data prevents conclusive evidence. It was also concluded that storing BPs in an airtight container of desiccant, under a dry nitrogen gas was the best way to prevent water retention overtime.
\end{abstract}

Keywords: Buckypaper, SWNT, FTIR, photothermal desorption

\section{Introduction}

The development of a method for fabricating carbon nanotubes (CNT) into self-supporting buckypapers (BPs) has been investigated for use in electronics[1,2], composite materials[3], and recently for volatile organic compound (VOC) sampling[4,5]. One of the most common fabrication methods is performed by suspending CNT in a surfactant-water solution, vacuum filtering against a membrane, and washing the resulting CNT cake with water to remove surfactants[2-6]. The inclusion of a water rinsing step is often thought to remove excess surfactants[6], but some studies have shown additional cleaning measures are needed[4,7]. Removal of surfactant is essential to increase conductivity[7] and maximize BP surface area by opening pores.

In our previous work, BPs were fabricated for use in air sampling and photothermal desorption (PTD)[4]. PTD is a novel desorption technique that uses broad spectrum light to directly heat BPs and desorb collected VOCs[8]. The concentration of desorbed VOC is then monitored by an inline detector such as a photo ionization detector or sent to a gas chromatograph for separation and quantitation[8]. BPs made for use with PTD were fabricated using arc discharge (AD) single-walled carbon nanotubes $(\mathrm{SWNTs})[5]$ dispersed in water with surfactant, vacuum filtered over a porous membrane, and cleaned with alternating acetone and deionized water rinses. Cleaned BPs were subject to an array of heat treatments, and it was determined that heating the $\mathrm{BPs}$ to $300^{\circ} \mathrm{C}$ for 90 minutes after rinsing was the preferred treatment to reduce surfactant load and increase surface area, while incurring minimal material loss from oxidation. Because non heat treated (NHT) BPs weighed more than their theoretical yield[5], the study concluded that heat treatment was necessary to fully remove surfactants. In previous studies[4,5] we used only gravimetric analysis to confirm BP purification. In the present work, the chemical composition of adsorbates on BPs was examined using Fourier-transform infrared spectrometry (FTIR). FTIR and Near-IR spectrometry have been utilized in many studies to characterize the chemical properties of CNT, however, most reports focus on raw CNTs[9,10], functionalized CNTs[1113], and BP composites[14,15]. Though FTIR has been used to investigate CNTs for materials usage, 
this paper is the first to use the spectrometric technique to determine the purity of BPs, an important quality assurance metric for sorbent materials.

\section{Methods}

\subsection{Buckypaper (BP) Fabrication}

Arc discharge (AD) SWNTs (94.5\% pure, 1.2-1.7 nm diameter, 0.1-4 $\mathrm{mm}$ length), pre-suspended in $1 \mathrm{mg} / \mathrm{mL}$ solution of $1 \% \mathrm{w} / \mathrm{v}$ sodium cholate $(\mathrm{SC})$ or sodium dodecyl sulfate (SDS) in water, was purchased from Nanointegris Inc. (Quebec, Canada). In accordance with the BP fabrication method suggested by the manufacturer, $50 \mathrm{~mL}$ of a $1 \mathrm{~g} / \mathrm{L}$ AD SWNT suspension was mixed with $400 \mathrm{~mL}$ of acetone for 13 hours. The suspended solution was then vacuum-filtered using a polytetrafluoroethylene (PTFE) membrane filter (47 mm diameter, $5 \mu \mathrm{m}$ pore size, EMD Milipore, Darmstadt, Germany). Immediately following filtration, the still wet SWNT cake underwent a series of two cleaning cycles; each cycle consisted of a rinse with $250 \mathrm{~mL}$ of deionized water $(18.2 \mathrm{M} \Omega \mathrm{cm})$, followed by a rinse with $40 \mathrm{~mL}$ of acetone (ACS grade). The SWNT cake was allowed to vacuum dry for 30 minutes, followed by 2 hours of air drying without vacuum. The resulting BP was delaminated from the PTFE membrane to form a self-supporting sorbent.

\subsection{Heat Treatment}

Heat treatment of BPs was performed using a muffle furnace (Thermolyne ${ }^{\mathrm{TM}}$-F48025-60-80, Thermo Fisher Scientific ${ }^{\mathrm{TM}}$, Waltham, MA) as previously reported[4]. Samples $(\mathrm{n}=3)$ were held at $300^{\circ} \mathrm{C}$ for 90 minutes in the furnace, using a ramping rate of $10^{\circ} \mathrm{C} / \mathrm{min}$, and allowed to cool to room temperature.

\subsection{Storage Effects on FTIR spectra}

To observe the effects of sample storage, three non-heat treated (NHT) BPs were subjected to various conditions immediately after fabrication. BP-1 was placed in a beaker of DrieRite®, covered with parafilm and stored at room temperature. BP-2 was placed into an air-tight jar with DrieRite®, purged with dry $\mathrm{N}_{2}$ gas and stored at $100^{\circ} \mathrm{C}$ in an oven. BP-3 was placed in an open container at $100^{\circ} \mathrm{C}$ in an oven according to the typical method[5]. Each BP was allowed to stay in its respective storage condition for 4 weeks, only disturbing them for weekly FTIR analysis. For the $\mathrm{N}_{2}$ stored $\mathrm{BP}$, the container was purged with $\mathrm{N}_{2}$ after each analysis, to keep the storage environment consistent.

\subsection{FTIR Characterization of BPs}

Using an attenuated total reflectance fourier-transform infrared spectrometer (ATR-FTIR) (ALPHA II Platinum ATR, Bruker ${ }^{\mathrm{TM}}$, Billerica, MA), with a single-reflection, diamond, optical crystal, BP spectra were collected for 3 samples before and after heat treatment so that the effects of heat treatment could be assessed. FITR Spectra were also obtained for each storage sample (i.e. BP 1-3) at one-week intervals over the course of a month (Week 1-4). Prior to and following each experimental scenario, the optical crystal and sample clamp were cleaned with ethanol to remove any residue. In addition, all BPs were placed directly placed onto the FTIR unit, with no additional sample preparations. The resulting spectra were then used to identify all moieties of interest, and the presence of residual fabrication materials was determined.

\section{Results and Discussion}

Transmissivity data was collected for $3 \mathrm{BPs}$, pre- and post-heat treatment, via FTIR (Figure 1). Peaks of interest for NHT BPs can be seen as the alkane stretch near $2900 \mathrm{~cm}^{-1}$ and the primary -OH stretch near $3400 \mathrm{~cm}^{-1}$. Though the fingerprint region of the spectra $\left(1500 \mathrm{~cm}^{-1}\right.$ to $\left.500 \mathrm{~cm}^{-1}\right)$ are obscured by water interference, these two stretches appear to be consistent with the spectra of both SDS and SC16, indicating the presence of remaining surfactant from SWNT suspension in the NHT samples. The 
spectra of for HT BPs have mostly been obscured by interference, however, there appears to be a carbonyl peak near $1700 \mathrm{~cm}^{-1}$, suggesting potential oxidation of SWNTs. Furthermore, the peaks of interest for NHT BPs, have decreased after heat treatment.

BPs Pre and Post HT
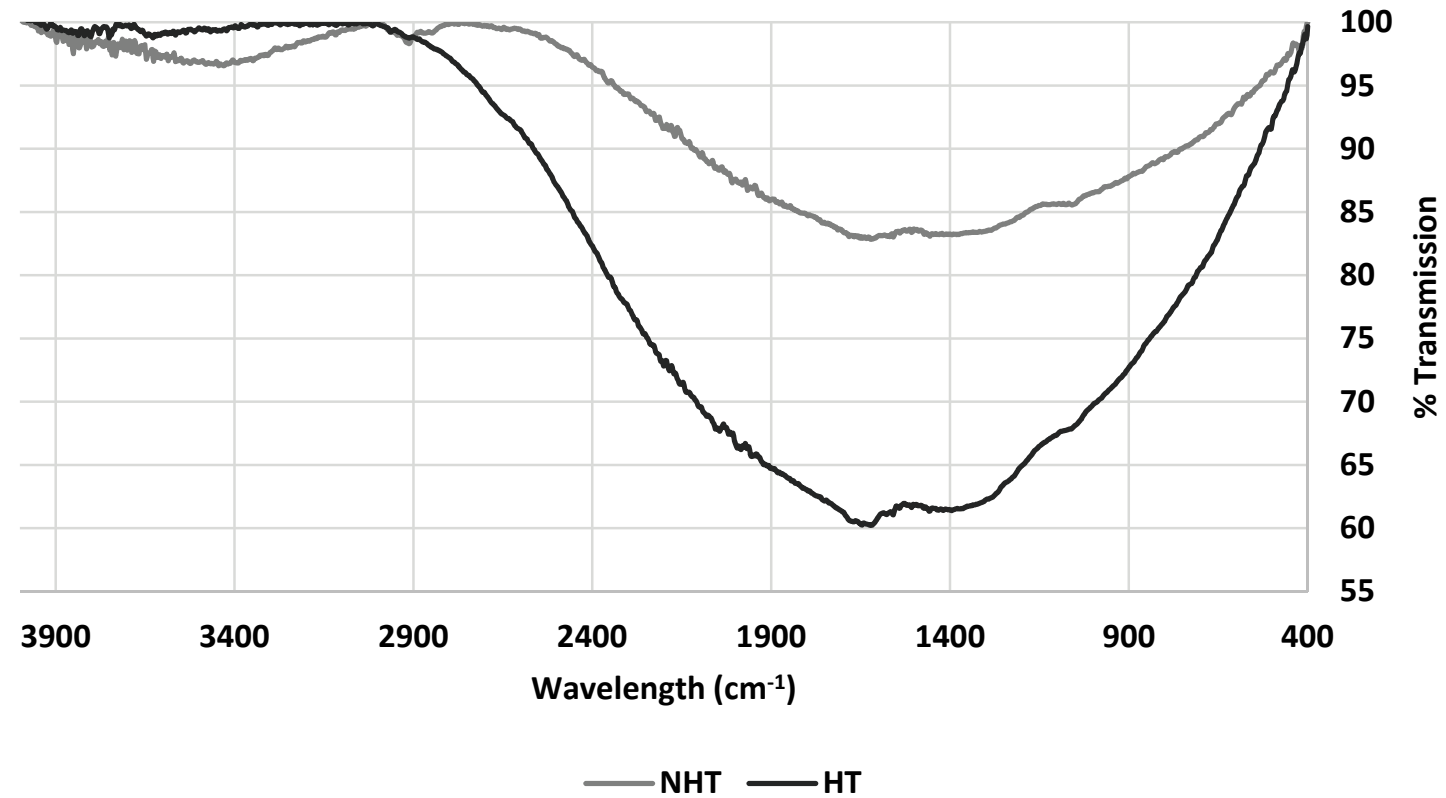

Figure 1. Comparison of the FTIR spectral data for triplicate BP samples before heat treatment (NHT) and after a 90 -minute heat treatment at $300^{\circ} \mathrm{C}$

Spectral data was collected for 3 NHT BPs stored in different conditions over four weeks (Figure 2). The absorption spectra labeled "initial" were collected immediately following fabrication (i.e. prior to storage). Differences observed in initial conditions are likely due to the lab environment on the time of fabrication and relative changes should be considered rather than absolute values. The idea of time variability is suggested by the differences in initial spectra of the DrieRite $(\mathrm{DR}) \mathrm{BP}$ and Oven $(\mathrm{O}) \mathrm{BP}$, considering they were fabricated on the same day. By Week 1, the interference on each BP was reduced. However, with each passing week the DR and O tend back to a similar level of interference as initial, while the $\mathrm{N}_{2}$ BP stays constant from Week 1 to 4 .

According to the study performed by Oh et al., when surfactants are removed from BPs during heat treatment, surface area increased by an average of $354 \%$ as adsorption sites were vacated[4]. Similarly, we observe a decrease in the peaks of interest for Figure 1, with an increase in interference, which suggests that heat treatment has been successful in removing majority of remaining surfactants. However, part of the alkane stretch $\left(2900 \mathrm{~cm}^{-1}\right)$ has been obscured by increased interference (indicated by decrease in $\%$ transmission), limiting the ability to conclusively state that all of the surfactant has been removed. This increase in interference is likely due to the presence of surface water which could be due to more available adsorption sites and surface oxidation that increases the affinity for water. The potential for surface oxidation is noted by an obscured carbonyl peak in Figure 1, though this peak is ultimately inconclusive due to a low signal to noise ratio. If surface oxidation has occurred, the hydrogen bonding capabilities of oxygen containing functionalities could further explain the increased affinity for water. Conversely, it has been reported that functionalities can decrease tube surface area[17], which conflicts with our previous findings[4]. It is possible that the $300^{\circ} \mathrm{C}, 90 \mathrm{~min}$ heat treatment causes enough surface oxidation to increase water affinity but not erode pore structures and reduce surface areas. With this in mind, we hypothesize that the increase in interference is primarily due to water adsorption likely related to the increase in available adsorption sites that have some surface oxidation. 
a) Dry-Rite ${ }^{\circledast}$ Storage $\left(24^{\circ} \mathrm{C}\right)$

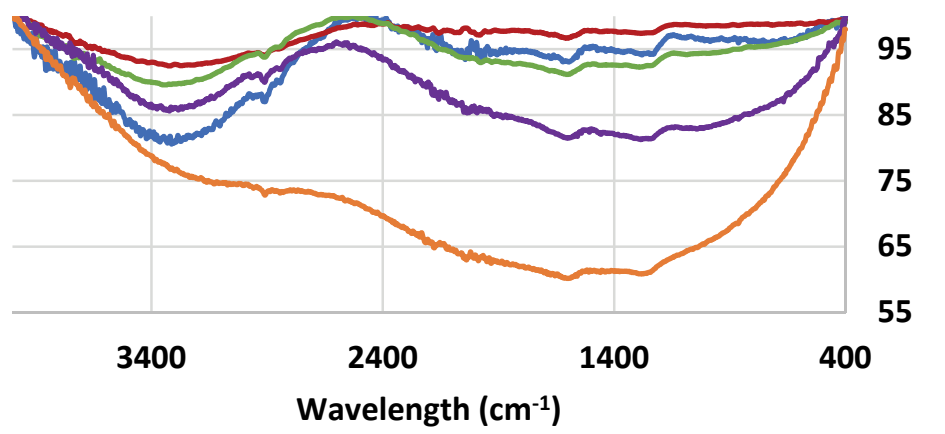

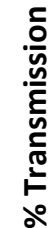

-Initial $\longrightarrow$ Week $1-$ Week $2-$ Week $3-$ Week 4

b)

Oven Storage $\left(100^{\circ} \mathrm{C}\right)$

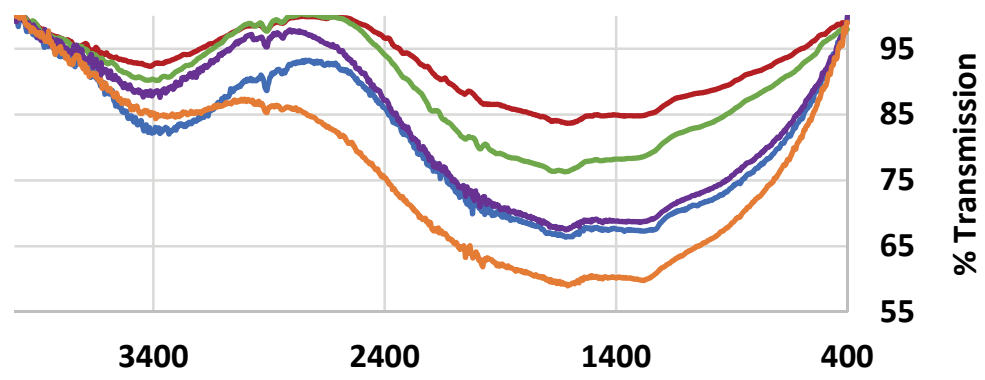

Wavelength $\left(\mathrm{cm}^{-1}\right)$

Initial $\longrightarrow$ Week $1-$ Week $2-$ Week $3-$ Week 4

c)

$\mathrm{N}_{2}$ Storage $\left(100^{\circ} \mathrm{C}\right)$

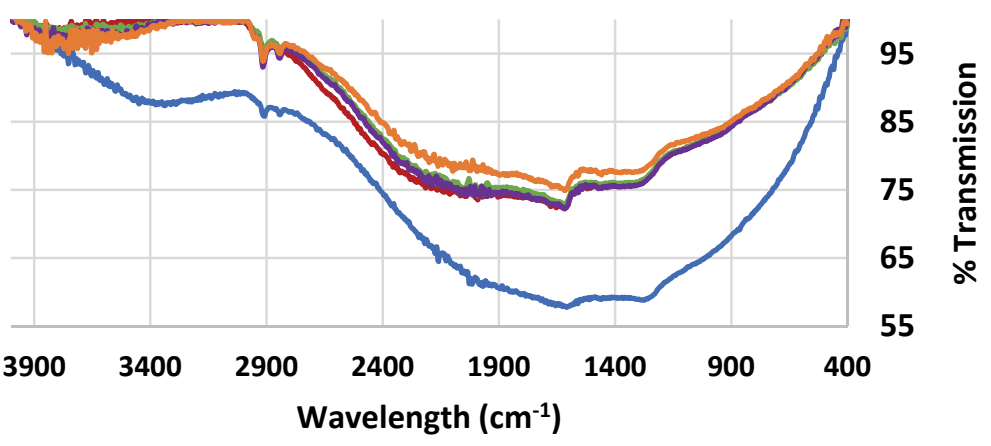

Initial —Week $1-$ Week $2-$ Week $3 \longrightarrow$ Week 4

Figure 2. FTIR spectra of varying storage methods at one-week intervals. A) Spectra of BP stored in air tight container of DrieRite ${ }^{\circledR}$. B) Spectra of BP stored uncovered in a $100^{\circ} \mathrm{C}$ oven. C) Spectra of BP stored at $100^{\circ} \mathrm{C}$ container of DrieRite® under dry $\mathrm{N}_{2}$ gas

All storage types (figure 2) were initially effective at removing some interference present in NHT samples. DrieRite ${ }^{\circledR}$ at room temperature appeared to be the most effective at reducing interference, but this particular sample had the least initial interference and the improvement was only retained for the 
first 2 weeks. This could have been due to the DrieRite ${ }^{\circledR}$ becoming saturated with water vapor. Oven storage at $100^{\circ} \mathrm{C}$ was initially effective but after 3 weeks of storage it too was the same-as or worse-than initial interference. $\mathrm{N}_{2}$ storage with DrieRite® and $100^{\circ} \mathrm{C}$ appeared to be the least effective at removing interference, but this sample showed the most initial interference of the three BPs tested and was clearly the most consistent across the study period. Degassing of the $\mathrm{N}_{2}$ storage sample removed the large interference bands but not the C-H stretching associated with the surfactant $\left(3900-3600 \mathrm{~cm}^{-1}\right)$. Though there was substantial difference in the initial interference of the three samples, it is reasonable to conclude that using an air tight container with desiccant and purged with dry $\mathrm{N}_{2}$ gas is the best storage method to preserve freshly prepared BP sorbents.

A potential limitation of the present work is the variability between each BP. Due to the fabrication method used (i.e. suspension and vacuum filtration), SWNTs are not necessarily deposited uniformly across the membrane filter, meaning that the thickness may vary across the material. Therefore, there is a potential for spectral differences across a single BP, based on the region of the BP measured.

\section{Conclusion}

This study set out to identify if FTIR could be used to quickly evaluate surfactant removal from buckypapers prepared from a surfactant aided SWNT suspension. FTIR analysis was able to capture a decrease in surfactant after heat treatment, as indicated by the reduction of signal between 3900 and $3600 \mathrm{~cm}^{-1}$. However, heat treatment seems to have caused a permanent increase in the absorption spectra that largely resembles the removable interference in non-heat-treated samples. This heat treatment absorption obscures surfactant absorption at $2900 \mathrm{~cm}^{-1}$ which leaves the less specific hydrocarbon stretches from $3900-3600 \mathrm{~cm}^{-1}$ to be used as the indicator of surfactant presence. FTIR analysis was suitable as a fast, qualitative check to confirm surfactant removal, but could not provide quantitative results correlated to previous studies. Along with determining the removal of surfactants, this study was able to determine that storage of BPs in an airtight jar with desiccant, and purging with dry $\mathrm{N}_{2}$ gas, is a satisfactory way to store freshly prepared or regenerated BP samples.

Conflicts of Interest. There are no conflicts to declare.

Acknowledgements. This study was supported by The Deep South Center for Occupational Health and Safety (Grant \#5T42OH008436-14) from the National Institute of Occupational Safety and Health; NIOSH), and by the North Carolina Occupational Safety and Health Education and Research Center (Grant \#T42OH008673), funded by NIOSH. Its contents are solely the responsibility of the authors and do not necessarily represent the official views of NIOSH.

\section{References}

1. Lu, S. et al. Fabrication of single/multi-walled hybrid buckypaper composites and their enhancement of electromagnetic interference shielding performance. J. Phys. D. Appl. Phys. 49, (2016).

2. Mustafa, I. et al. Fabrication of Freestanding Sheets of Multiwalled Carbon Nanotubes (Buckypapers) for Vanadium Redox Flow Batteries and Effects of Fabrication Variables on Electrochemical Performance. Electrochim. Acta 230, 222-235 (2017).

3. Khan, Z. U., Kausar, A., Ullah, H., Badshah, A. \& Khan, W. U. A review of graphene oxide, graphene buckypaper, and polymer/graphene composites: Properties and fabrication techniques. J. Plast. Film Sheeting 32, 336-379 (2016).

4. Oh, J., Floyd, E. L., Parit, M., Davis, V. A. \& Lungu, C. T. Heat Treatment of Buckypaper for Use in Volatile Organic Compounds Sampling. J. Nanomater. 2016, (2016).

5. Oh, J., Floyd, E. L., Watson, T. C. \& Lungu, C. T. Fabrication and adsorption characterization of single-walled carbon nanotubes (SWNT) buckypaper (BP) for use in air samples. Anal. Methods 8, 4197-4203 (2016).

6. Wu, Z. Transparent, Conductive Carbon Nanotube Films. Science (80-. ). 305, 1273-1276 (2004).

7. Wang, J. et al. Removal of the Residual Surfactants in Transparent and Conductive Single Walled Carbon Nanotube Films.pdf. 17685-17690 (2009). 
8. Floyd, E. L., Sapag, K., Oh, J. \& Lungu, C. T. Photothermal Desorption of Single-Walled Carbon Nanotubes and Coconut Shell- Activated Carbons Using a Continuous Light Source for Application in Air Sampling. Ann. Occup. Hyg. 58, 877-888 (2014).

9. Itkis, M. E. et al. Purity evaluation of as-prepared single-walled carbon nanotube soot by use of solution-phase near-IR spectroscopy. Nano Lett. 3, 309-314 (2003).

10. Sbai, K. et al. Infrared spectroscopy of single-walled carbon nanotubes. J. Phys. Chem. B 110, 12388-93 (2006).

11. Bazuła, P. A., Lu, A. H., Nitz, J. J. \& Schüth, F. Surface and pore structure modification of ordered mesoporous carbons via a chemical oxidation approach. Microporous Mesoporous Mater. 108, 266-275 (2008).

12. Spinato, C. et al. Different chemical strategies to aminate oxidised multi-walled carbon nanotubes for siRNA complexation and delivery. J. Mater. Chem. B 4, 431-441 (2016).

13. Wepasnick, K. A. et al. Surface and structural characterization of multi-walled carbon nanotubes following different oxidative treatments. Carbon N. Y. 49, 24-36 (2011).

14. Varga, M. et al. Diamond/carbon nanotube composites: Raman, FTIR and XPS spectroscopic studies. Carbon N. Y. 111, 54-61 (2017).

15. Martinez-Rubi, Y. et al. Tailored SWCNT functionalization optimized for compatibility with epoxy matrices. Nanotechnology 23, (2012).

16. National Institute of Advanced Industrial Science and Technology. SDBSWeb.

17. Ren, X., Chen, C., Nagatsu, M. \& Wang, X. Carbon nanotubes as adsorbents in environmental pollution management: A review. Chem. Eng. J. 170, 395-410 (2011). 